\title{
FREQUENCY COMB GENERATION IN A NONLINEAR RESONATOR THROUGH MODE COUPLING USING A SINGLE TONE DRIVING SIGNAL
}

David A. Czaplewski ${ }^{1}$, Steven W. Shaw ${ }^{2}$, Oriel Shoshani ${ }^{3}$, Mark I. Dykman ${ }^{4}$, and Daniel Lopez ${ }^{1}$

${ }^{1}$ Argonne National Laboratory, Argonne, IL, USA

${ }^{2}$ Florida Institute of Technology, Melbourne, FL, USA

${ }^{3}$ Ben-Gurion University of the Negev, Beersheba, ISR

${ }^{4}$ Michigan State University, East Lansing, MI, USA

\begin{abstract}
In this paper, we demonstrate bursting behavior in a nonlinear microelectromechanical (MEMS) resonator that creates a frequency comb in the corresponding spectral response. The bursting behavior occurs for a single driving tone applied to the resonator. The bursting behavior arises from the non-linear analog of "level anti-crossing" in a 1:3 internal resonance that can efficiently transfer energy between two modes of a resonator at low excitation amplitudes. The internal resonance creates a region in parameter space where stable oscillations do not exist, resulting in a forbidden zone of operation.
\end{abstract}

\section{INTRODUCTION}

Mode coupling generates many interesting and novel behaviors in mechanical [1], optical [2], quantum information [3], and biological [4] systems. When the frequencies of two modes are integer multiples of each other, an internal resonance can be achieved, resulting in efficient inter-modal energy transfer. Resonant mode coupling in closed loop applications has led to novel system behaviors including increased frequency stabilization [5] and the appearance of coherence energy transfer between modes, where the amplitude of the oscillations does not decay for a given period of time after the system drive has been turned off [6].

In this paper, we study resonant mode coupling in a nonlinear microelectromechanical (MEMS) resonator using an open loop configuration with a single drive frequency. Open loop driving allows us to explore the dynamic response of the system at and near internal resonance at different levels of the drive. We identified two new dynamic behaviors: a bursting type behavior, where the response of the resonator changes drastically on a timescale 5 orders of magnitude greater than the period of a single oscillation, and a "forbidden zone", where the resonator does not exhibit stable operations due to the continual exchange of energy between modes. The bursting behavior results from a bifurcation in the response caused by the internal resonance. We demonstrate control over the bursting period and associated spectrum with a single parameter, the detuning of the drive frequency from the internal resonance frequency.

\section{EXPERIMENT AND RESULTS}

We study the non-linear dynamic of a MEMS resonator fabricated from an SOI wafer using the SOIMUMPS process from MEMSCAP. The resonator consists of three clamped-clamped single crystal silicon beams connected in the center to two comb drives (Fig. 1a). The resonator is actuated electrostatically through the output of a Zurich UHFLI lock-in amplifier through one comb drive while the movement is sensed via the displacement current generated in the opposing comb drive that is converted into a voltage through a transimpedance amplifier (FEMTO DHPCA-200) and recorded using the lock-in amplifier.

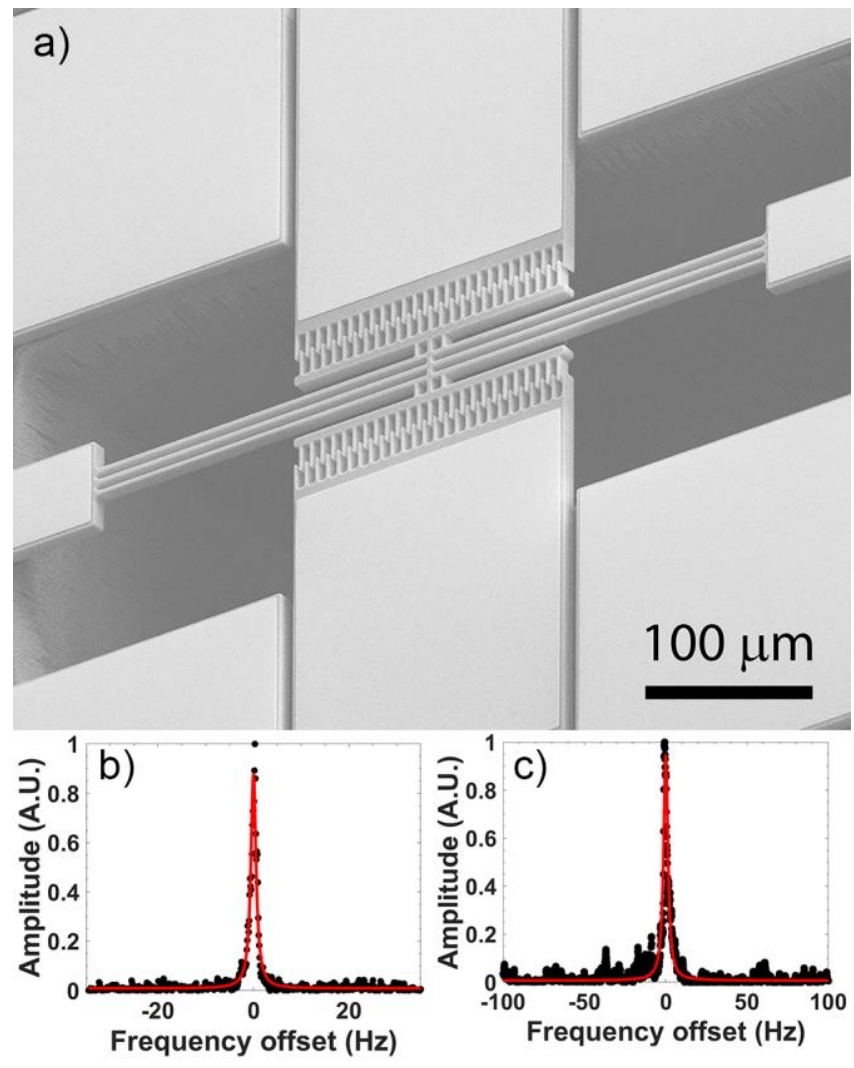

Figure 1 (a) Scanning electron micrograph of the MEMS resonator showing three single crystal silicon beams connected at their center. The device is actuated using one of the comb drive actuators while the motion is sensed through the generation of a motional current by the other comb. (b) Graph of the amplitude response of the flexural mode as a function of drive frequency offset from the peak response. (c) Graph of the amplitude response of the torsional mode as a function of drive frequency offset from the peak response.

The frequency response of the MEMS is recorded for various drive voltages. Using the lock-in, the drive amplitude is fixed and the frequency of the drive signal is increased from below the linear resonance frequency of the flexural mode to well above it. The response of the resonator to an applied voltage of $100 \mu \mathrm{V}$ can be seen in Fig. $1 \mathrm{~b}$ and corresponds to the fundamental in-plane flexural mode [5]. The response is fit to a Lorentz function and the frequency is measured to be $64580 \mathrm{~Hz}$ and the spectral linewidth of the resonator at half power is $0.6 \mathrm{~Hz}$. Similarly, the response of 
a)
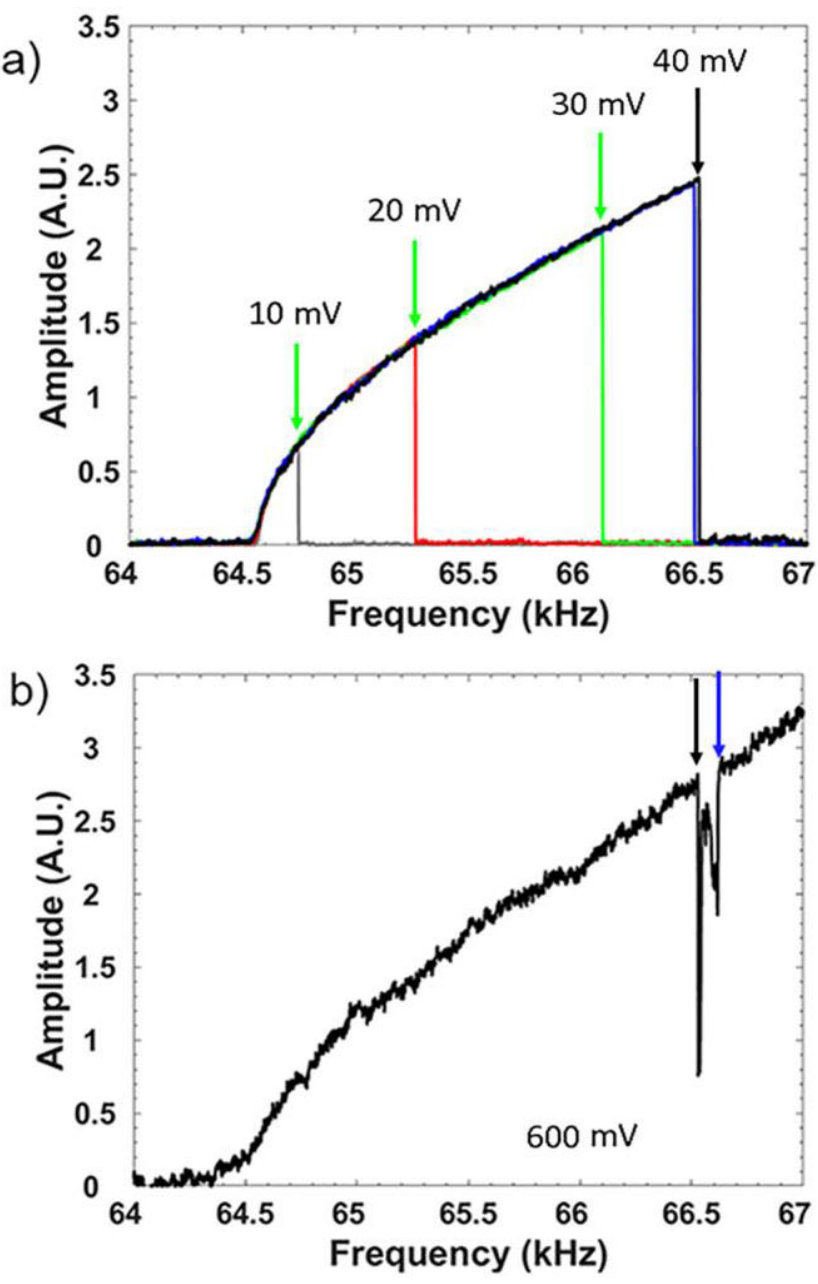

c)

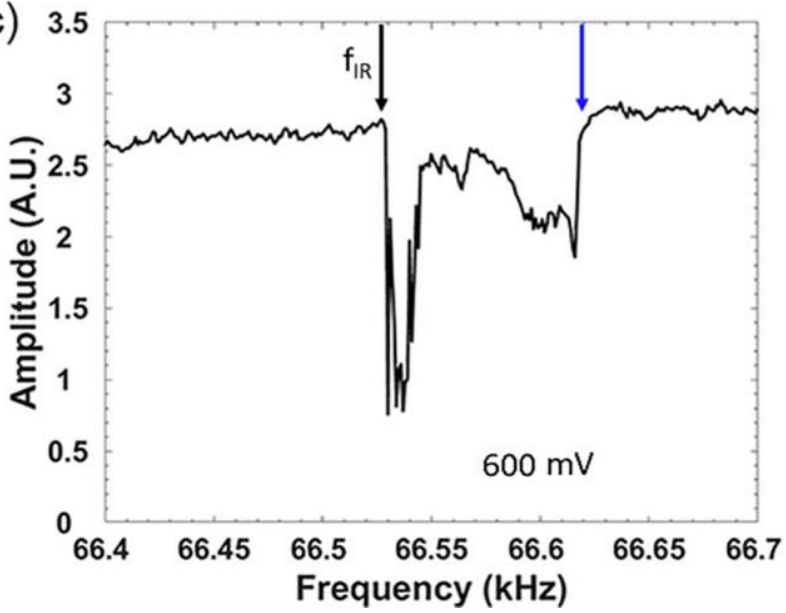

Figure 2 Amplitude of the first harmonic of the response, i.e., the flexural mode, of the resonator plotted versus the drive frequency. a) As the drive amplitude is increased, the frequency at which the resonator falls to the lower response branch increases until reaching the internal resonance frequency, at which the transitions to the lower branch accumulate. b) For large enough drive amplitudes, the response does not fall to the lower branch, but becomes modulated until resuming normal Duffing behavior. c) Magnified response near internal resonance showing deviation from Duffing behavior that leads to lower average amplitude, but does not transition to the lower branch.
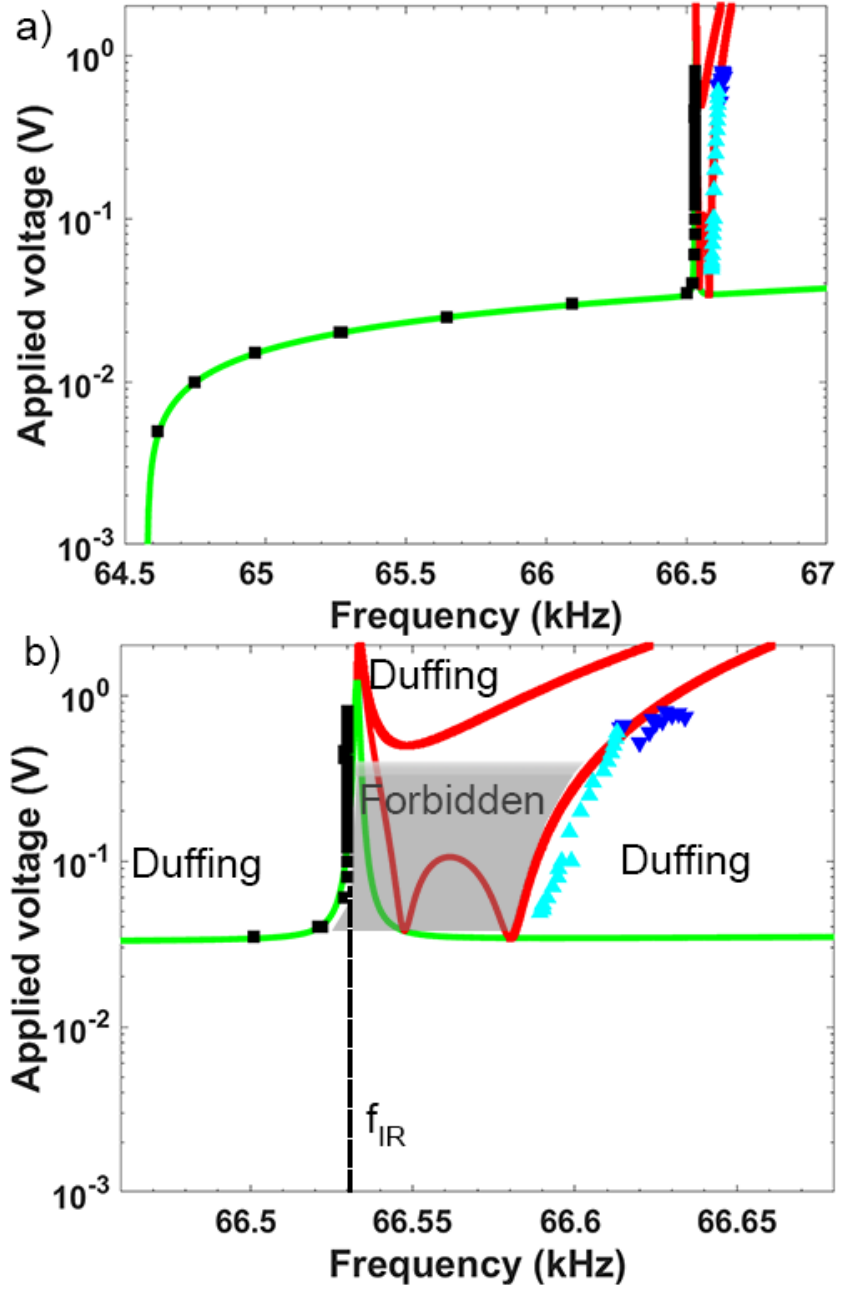

Figure 3 Bifurcation conditions for which the resonator response changes from Duffing behavior shown in the applied drive voltage versus the drive frequency plane. The squares and triangles are the experimental data while the solid lines are predictions from a model [7, 8].

a high frequency mode, corresponding to an out-of-plane torsional oscillation [5], is measured (Fig. 1c) at an applied voltage of 500 $\mathrm{mV}$ and the frequency found to be $199599 \mathrm{~Hz}$ with a spectral line width of $1.6 \mathrm{~Hz}$. The drive amplitude required to actuate the torsional mode is significantly higher than that of the flexural mode due to the inefficient coupling of the drive signal to the torsional degree of freedom using comb drive actuators [5].

As the frequency is swept around the flexural mode resonance at increasingly larger drive voltages, the response of the resonator deviates from Lorentzian and begins to exhibit asymmetries, indicative of a hardening Duffing response [5]. With further increase in drive amplitude, the response curve becomes bistable at larger frequencies, where the resonator response will ultimately fall from the upper branch of the stable response curve to the lower branch. As seen in Fig 2a, the frequency at which this occurs (Fig. 2a green arrows) continues to increase with driving amplitude, until it saturates when it reaches a value around $1 / 3$ of the resonance frequency of the torsional mode, as indicated by a black arrow in Fig. 2a. This behavior is indicative of an internal resonance, where the frequency of one mode corresponds with an integer multiple of the frequency of another mode. As the drive 
amplitude is increased, the frequency at which the response falls to the lower branch remains constant at the beginning of internal resonance. However, for even larger drive amplitudes, the response of the resonator can pass through the internal resonance frequency without falling to the lower branch (Fig. 2b black arrow) and finally resume expected Duffing behavior (Fig. 2b blue arrow) after a finite frequency interval. Fig. $2 \mathrm{c}$ shows that for this finite frequency interval, the time averaged amplitude is below the expected response from the Duffing model. As the drive amplitude is increased, the range of frequencies over which this reduced mean amplitude behavior occurs, increases. Instead of showing multiple response plots of the data in Fig. 2, it is convenient to plot the frequencies where the resonator response bifurcates, or changes significantly, as a function of the drive amplitude. This mapping from the behavior depicted in Fig. 2 to a bifurcation diagram is shown in Fig. 3. The black squares in Fig. 3a represent the frequencies at which the saddle node bifurcation (Duffing bifurcation) is observed. When the drive amplitude is insufficient to achieve the internal resonance frequency (Fig. 2a green arrows), the resonator response changes from the upper branch to the lower branch at the saddle node bifurcations resulting from the Duffing response as indicated by the vertical lines. For larger drive amplitudes, the resonator response deviates from Duffing behavior and shows a reduced mean behavior starting at the onset of internal resonance (Fig. 2c black arrow) and resumes Duffing behavior again (Fig. 2c blue arrow) at higher frequencies, marked by dark blue triangles in Fig. 3a. The creation of the bifurcation diagram in Fig $3 \mathrm{a}$ via frequency sweeps at varying drive levels identifies the locations of many of the system bifurcations. In order to further characterize the bifurcation diagram and determine the resonator response on the right hand side, the resonator was first driven with large enough amplitude to achieve frequencies greater than the internal resonance condition and resume normal Duffing behavior. The drive amplitude was then reduced and the drive frequency decreased toward the internal resonance. When the resonator approaches internal resonance, it transitions to the lower branch as shown in Fig. 3a as light blue triangles. Using this method, the hysteretic frequency response of the resonator is characterized for all frequencies around the internal resonance.

The graph in Fig. $3 b$ shows a zoomed-in region of the parameter space shown in Fig. 3a. The resonator response shows Duffing behavior for much of the operating space, as indicated by the labels "Duffing." When the resonator is driven to those regions of operation, the internal resonance condition is not evident, only the Duffing behavior is measured. However, for the region of internal resonance and low drive levels, the resonator does not have stable oscillations. There is a region of parameter space that is "forbidden", where the resonator transitions to the lower branch of operation and no stable oscillation can be maintained. For driving amplitudes larger than the forbidden region (shaded area in Fig. $3 \mathrm{~b}$ ), the mean amplitude of the resonator is smaller than the expected Duffing response, as shown in Fig. 2c.

A novel dynamic behavior is observed for large driving amplitudes above the forbidden zone. Fig. 4a shows the response of the resonator as a function of time when driven by a single frequency drive. It can be seen from the graph (Fig. 4a blue line) that the response of the first harmonic does not have a constant amplitude: it shows bursting behavior, i.e. it oscillates with a nonsinusoidal pattern which repeats in time with a period several orders of magnitude longer than the relaxation time of either mode. Simultaneous measurement of the third harmonic of the response shows similar periodic behavior.
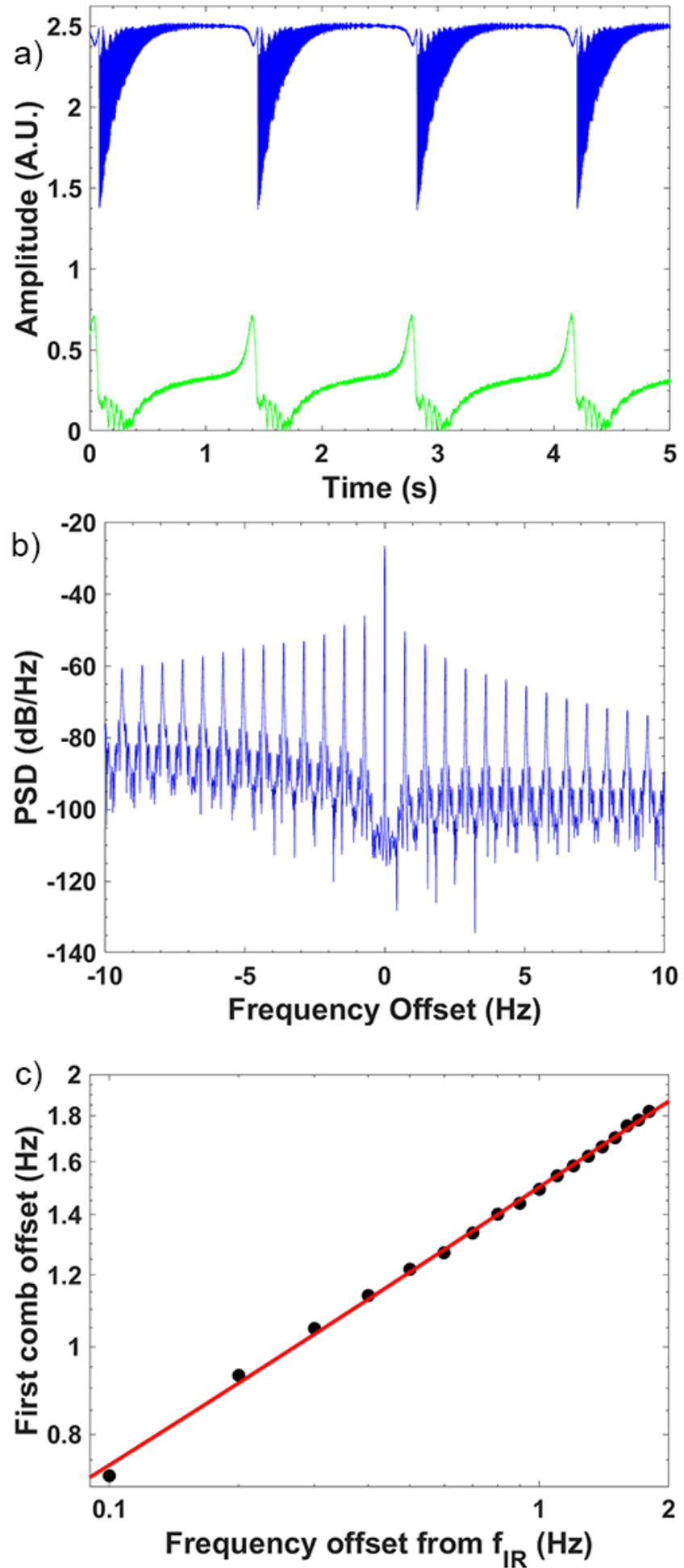

Figure 4 a) Amplitude of the first harmonic (blue) and third harmonic (green) components of the response of the resonator to a harmonic drive inside the internal resonance region. b) Fast Fourier Transform of the first harmonic of the response in (a) showing the generation of a frequency comb with consistent spacing between the combs. c) Position of the first comb in the FFT as a function of offset frequency from the internal resonance showing cubic scaling (red line). 
At internal resonance, the modes typically associated with the first harmonic (flexural) and third harmonic (torsional) undergo a hybridization [9] and no longer can be described by purely flexural or purely torsional motion in the degrees of freedom. An FFT analysis of the first harmonic component of the response can be seen in Fig. 4b. The bursting nature of the response creates a frequency comb in the spectrum with equally spaced combs in spite of the fact that the resonator is driven with a single frequency drive. As the drive frequency is increased across the internal resonance, the period of the bursting (Fig. 4a) decreases and the spacing of the combs increases. A similar result is obtained from the third harmonic. A graph of the comb spacing versus offset frequency from the internal resonance can be seen in Fig. 4c. A fit of the data to the log-log plot has a slope of $1 / 3$, indicating a cubic relationship between the comb spacing and the driving frequency. The measured response is repeatable with respect to the drive frequency. Also, the response does not show any hysteresis with regards to the frequency; the period of motion and subsequent frequency comb spacing is completely determined by the frequency offset from the onset of internal resonance.

\section{CONCLUSIONS}

In this paper, we demonstrate a non-linear MEMS resonator whose primary flexural mode can be detuned through large amplitude driving to achieve mode coupling with the primary torsional mode at an internal resonance. We build a bifurcation diagram of the response of the resonator in the plane of the drive parameters. Near the internal resonance, our measurements demonstrate a region of phase space where the resonator does not show stable oscillations, creating a "forbidden zone" where stable operation is not possible. For single frequency driving signals with amplitudes greater than the forbidden zone, the resonator dynamics is characterized by bursting behavior resulting in the generation of a frequency comb. We speculate that the bifurcation causing the bursting behavior demonstrated here is similar to the mechanism for spiking in neuron models, making this resonator structure a possible candidate to study the mechanical analog of interacting neurons [10].

\section{ACKNOWLEDGEMENTS}

The authors thank Scott Strachan for help with the bifurcation diagram. This work was performed, in part, at the Center for Nanoscale Materials, a U.S. Department of Energy Office of Science User Facility, and supported by the U.S. Department of Energy, Office of Science, under Contract No. DE-AC0206CH11357. OS acknowledges partial support from Ben-Gurion University of the Negev. SWS acknowledges support for this work from the US National Science Foundation under grants CMMI1662619 and CMMI-1561829.

\section{REFERENCES}

[1] R. DeAlba, F. Massel, I. Storch, T. Abhilash, A. Hui, P. McEuen, H. Craighead, and J. Parpia, Nature Nanotechnology 11, 741 (2016).

[2] A. Sharin, N. Flowers-Jacobs, S. Hoch, A. Kashkanova, C. Deutsch, J. Reichel, and J. Harris, Physical Review Letters 112, 013602 (2014).

[3] E. Veragen, S. Deleglise, S. Weis, A. Schliesser, and T. J. Kippenberg, Nature 482, 63 (2012).

[4] G. Buzsaki and A. Draguhn, Science 304, 1926 (2004).

[5] D. Anonio, D. Zanette, and D. Lopez, Nature Comm. 3806 (1012).

[6] C. Chen, et al., Nat. Comm. 815523 (2017).

[7] O. Shshani, S. W. Shaw, and M. I. Dykman, Scientific Reports 718091 (2017).

[8] Scott Strachan, Ph.D. dissertation, Department of Mechanical Engineering, Michigan State University (2017).

[9] J. Guttinger, A. Noury, P. Weber, A. M. Eriksson, C. Lagoin, J. Moser, C. Eichler, A. Wallraff, A. Isacsson, and A. Bachtold, Nature Nano. 12, 631 (2017).

[10] P. De Maesschalck and M. Wechselberger, J. of Math. Neuroscience 516 (2015).

\section{CONTACT}

*D.A. Czaplewski, tel: +1-630-252-3258; dczaplewski-at-anl.gov 